\title{
Effects of decomposition-treatment temperature on infiltration pressure of a surface modified nanoporous silica gel
}

\author{
Aijie Han, Venkata K. Punyamurtula, Yu Qiao* \\ Department of Structural Engineering, University of California, San Diego, La Jolla, CA 92093-0085, USA \\ Received 22 September 2007; received in revised form 12 November 2007; accepted 4 December 2007
}

\begin{abstract}
When a nanoporous silica gel surface modified by silyl groups is decomposition-treated at a relatively low temperature, while the network is stable, the organic surface layers can be deactivated. As a result, the degree of hydrophobicity, which can be measured by the liquid infiltration pressure, is lowered. The infiltration and defiltration behaviors of liquid are dependent on the controlled decomposition-treatment time, the liquid composition, as well as the testing temperature. By adding electrolyte or using higher testing temperature, the infiltration pressure can be increased and the defiltration can be promoted.
\end{abstract}

(C) 2008 Elsevier B.V. All rights reserved.

Keywords: Nanoporous; Silica; Surface treatment

\section{Introduction}

Nanoporous materials are being widely used for biological, chemical, and environmental engineering applications [1]. Recently, their application was extended to mechanical systems, e.g. energy absorption systems [2,3]. The working mechanism is associated with the behaviors of pressurized liquids in nanoenvironment. The system consists of a liquid phase, usually water of an aqueous solution, and nanoporous particles suspended in it. The nanopore surface should be hydrophobic, so that it is energetically unfavorable for water to enter into the nanopores. A sufficiently high external pressure must be applied to overcome the energy barrier caused by the hydrophobicity of the pore walls. As the pressurized liquid enters into the nanopores, the solid and liquid contact each other over the large nanopore surface. Thus, the free energy of the system increases significantly. The increase in free energy is $\Delta U=\Delta \gamma \cdot A$, where $\Delta \gamma$ is the excess solid-liquid interfacial energy and $A$ is the specific surface area. Typically, $\Delta \gamma$ is $10-50 \mathrm{~mJ} / \mathrm{m}^{2}$ [4]. If the pore size, $r$, is at the $\mu \mathrm{m}$ level, the specific surface area, $A$, is around a few $\mathrm{m}^{2} / \mathrm{g}$, and thus the energy density, $\Delta U$, is $100-500 \mathrm{~mJ} / \mathrm{g}$, comparable with that of conventional energy absorption materials, such as shape memory alloys and reinforced polymers

\footnotetext{
* Corresponding author.

E-mail address: yqiao@ucsd.edu (Y. Qiao).
}

[5]. If the pore size is $1-10 \mathrm{~nm}$, the specific surface area can be $100-1000 \mathrm{~m}^{2} / \mathrm{g}$, and therefore $\Delta U$ is $1-50 \mathrm{~J} / \mathrm{g}$, attractive for developing lightweight and small-sized protective and damping devices, e.g. soldier armors and vehicle bumpers.

During the past a few decades, a number of nanoporous materials have been developed [6,7], among which nanoporous silicas are suitable to mechanical applications. Their nanopore size can be controlled in the range of $2-100 \mathrm{~nm}$ and therefore their specific surface areas are around $100-1000 \mathrm{~m}^{2} / \mathrm{g}$ [8]. The nanoporous structure is quite stable even under a high pressure of around $500 \mathrm{MPa}$, sufficient for most of engineering applications. However, silica is intrinsically hydrophilic, and consequently an unmodified nanoporous silica gel can be soaked by water spontaneously. To trigger pressure-induced infiltration, the inner surfaces of nanopores must be covered by hydrophobic groups, very often silyl groups.

For a protective or damping system, the working pressure must be adjusted in specified ranges. On the one hand, it must be smaller than the safety point, so that unexpected damages would not occur. On the other hand, it should be as high as possible, so as to maximize the energy absorption efficiency. In our previous experimental studies, it was noticed that the degree of hydrophobicity, which dominates the infiltration pressure, is related to the surface coverage of silyl groups, $c[9,10]$. As the density of hydrophobic groups at nanopore surface increases, the infiltration pressure becomes higher, until the surface coverage saturates. The value of $c$ can be controlled quite precisely by 
surface treating nanoporous silica for different periods of time. However, such a technique may not work well if the required surface group density is relatively low. If the treatment time is too short, the diffusion of silyl molecules inside nanopores cannot reach the equilibrium condition, and the surface coverage may be nonuniform along the axial direction. In the current study, we investigate another method for control of surface coverage: decomposition-treatment. After a saturated surface group layer is produced, its density can be lowered by heating the material. Since the decomposition process is directly related to the temperature field and the effects of molecular diffusion should be secondary, the change in surface properties tends to be homogeneous.

\section{Experimental}

An Aldrich-60758 silica gel modified with chloro (dimethyl)octadecylsilane $\left(\mathrm{C}_{18}\right)$ was investigated. By using a Tristar-3000 gas absorption analyzer, the average nanopore diameter has been found to be $7.0 \mathrm{~nm}$, and the specific surface area was $234 \mathrm{~m}^{2} / \mathrm{g}$. The particle size was $20-50 \mu \mathrm{m}$.

The end-capping modification was performed in a roundbottom flask. The silica gel was first dried at $100^{\circ} \mathrm{C}$ for $24 \mathrm{~h}$ in vacuum, and then immersed in $2.5 \%$ dry toluene solution of chlorotrimethylsilane $\left(\mathrm{C}_{1}\right)$. The mixture was stirred at room temperature $\left(20^{\circ} \mathrm{C}\right)$ for $10 \mathrm{~min}$, and then refluxed at $90^{\circ} \mathrm{C}$ for 5 days. During this process, the chlorotrimethylsilane molecules diffused into the nanopores and deactivated the negatively charged hydroxyl groups, forming $\mathrm{OSi}\left(\mathrm{CH}_{3}\right)_{3}$ groups [11]. Due to the relatively long treatment time, the surface group density was saturated; that is, further increase in treatment time would not lead to detectable variation in the degree of hydrophobicity [12]. The surface-treated silica gel was collected in a vacuum filter, washed by dry toluene and warm water for a few times, and dried in vacuum at $100{ }^{\circ} \mathrm{C}$ for $5 \mathrm{~h}$. The average nanopore diameter of the surface-treated silica gel has been found to be $6.0 \mathrm{~nm}$, and the specific surface area was $219 \mathrm{~m}^{2} / \mathrm{g}$. The material was then placed in a tube furnace and heated at $180{ }^{\circ} \mathrm{C}$. The treatment time ranged from $6 \mathrm{~min}$ to $1.5 \mathrm{~h}$. The setpoint of $180^{\circ} \mathrm{C}$ was chosen because a higher or lower temperature would lead to a reduced controllability of treatment time. An air flow was maintained during the decompositiontreatment.

The surface property of the decomposition-treated silica gel samples was examined through a pressure-induced liquid infiltration experiment. The testing cell contained $0.4 \mathrm{~g}$ of decomposition-treated silica gel and $6 \mathrm{~g}$ of liquid sealed in a stainless steel cylinder. The liquid phase was either distilled water or saturated sodium chloride solution. As the nanopore surface was hydrophobic, the liquid does not enter the nanopores spontaneously, and the initial system volume was the summation of the volumes of the two components. An external pressure was applied through a piston by using a type 5569 Instron machine. The piston was compressed into the steel cell at a constant rate of $1 \mathrm{~mm} / \mathrm{min}$. As the pressure reached about $50 \mathrm{MPa}$, the piston was moved back at the same rate. During the experiment, the temperature of the testing cell was kept by a water bath either

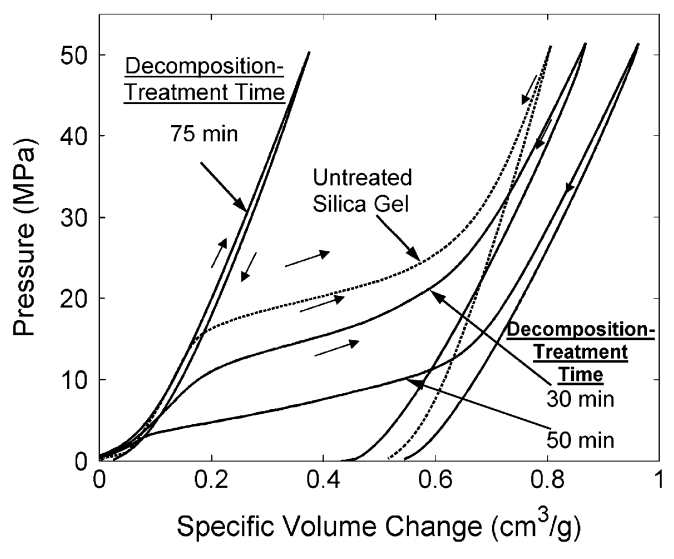

Fig. 1. Typical sorption isotherm curves at room temperature.

at room temperature $\left(20^{\circ} \mathrm{C}\right)$ or at $85^{\circ} \mathrm{C}$. The typical sorption isotherm curves are shown in Figs. 1 and 2.

\section{Results and discussion}

The dashed line in Fig. 1 shows the behavior of the surfacemodified silica gel without any decomposition-treatment. As the nanopore surface is decorated by silyl groups, it becomes hydrophobic, and thus the liquid phase does not enter the nanopores until the pressure is sufficiently high. As the liq-
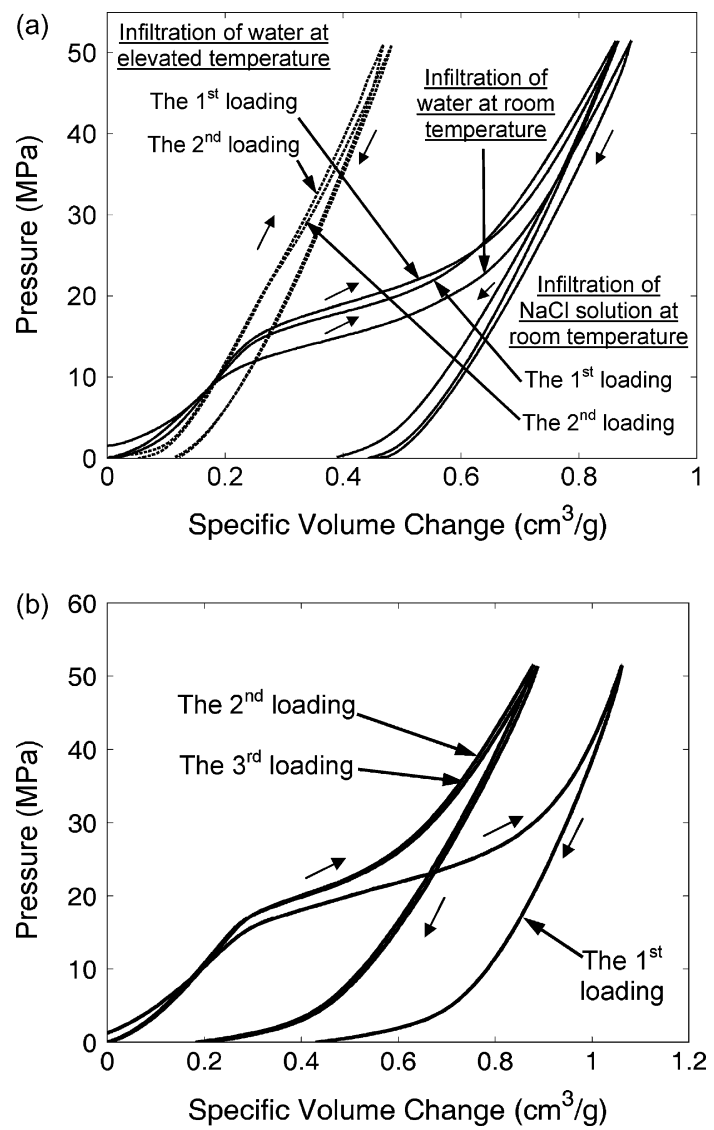

Fig. 2. Typical sorption isotherm curves of silica gel that is decompositiontreated for $30 \mathrm{~min}$ : (a) with sodium chloride solution or at elevated temperature; (b) with sodium chloride solution and at elevated temperature. 
uid starts to enter the nanopores, the system volume largely decreases with a relatively small pressure increase. When the nanopores are filled, the system volume is reduced from $V_{\mathrm{s}}+V_{1}$ to $V_{\mathrm{s}}+V_{1}-V_{\mathrm{p}}$, with $V_{\mathrm{s}}, V_{1}$, and $V_{\mathrm{p}}$ being the volumes of silica gel, liquid phase, and nanopores, respectively. The rapid change in volume causes the formation of the infiltration plateau in sorption isotherm curve around $22 \mathrm{MPa}$. In the following discussion, the infiltration pressure, $p_{\text {in }}$, is taken as the pressure at the middle point of the infiltration plateau. By using the classic Laplace-Young equation [13], the associated solid-liquid interfacial tension can be estimated as $\Delta \gamma=p_{\text {in }} \cdot d / 4=41 \mathrm{~mJ} / \mathrm{m}^{2}$. Note that the liquid infiltration in nanopores is an irreversible process. As the pressure is reduced, the sorption isotherm curve is quite linear, indicating that the confined liquid does not defiltrate.

As the silica gel was decomposition-treated for $30 \mathrm{~min}$ at $180^{\circ} \mathrm{C}$, the major characteristics of the sorption isotherm curve remain similar, while the infiltration pressure is reduced to $16 \mathrm{MPa}$. Correspondingly, the effective solid-liquid interfacial energy is $\Delta \gamma=p_{\text {in }} \cdot d / 4=30 \mathrm{~mJ} / \mathrm{m}^{2}$, decreased by nearly $25 \%$. Clearly, during the decomposition-treatment, a certain portion of the organic surface groups decompose, i.e. less nanopore wall area is covered by hydrophobic layer, resulting in the decrease in the degree of hydrophobicity. Note that the width of infiltration plateau increases slightly by about $6 \%$. The length of $\mathrm{OSi}\left(\mathrm{CH}_{3}\right)_{3}$ surface group is around $0.4 \mathrm{~nm}$. In a nanopore of the diameter of $7.0 \mathrm{~nm}$, a surface layer with the thickness of $0.4 \mathrm{~nm}$ occupies nearly $20 \%$ of the total volume. If $20-30 \%$ of the surface layer were decomposed, the infiltration volume would increase by around $6 \%$. This estimation is in agreement with the experimental result of infiltration pressure variation. The slope of infiltration plateau also increases slightly, indicating that the effective nanopore size distribution is broader. The decrease in infiltration pressure at the low-pressure end, i.e. in larger nanopores, is more pronounced compared with that at the high-pressure end, i.e. in smaller nanopores, probably because that the larger nanopores are of smaller specific surface area and tend to be more opened to the environment.

As the decomposition-treatment time increases to $50 \mathrm{~min}$, the infiltration pressure further decreases to $7.6 \mathrm{MPa}$, and the solid-liquid interfacial energy is reduced to $14 \mathrm{~mJ} / \mathrm{m}^{2}$, smaller than that of untreated material by nearly $2 / 3$. Compared with the silica gel treated for $30 \mathrm{~min}$, the slope and the width of infiltration plateau increase slightly, suggesting that the surface groups are further decomposed. That is, the degree of hydrophobicity is lower; the accessible nanopore volume is larger; and the effective nanopore size distribution is broader.

The relationship between the infiltration pressure, $p_{\text {in }}$, and the treatment time, $t_{\mathrm{d}}$, is more clearly shown in Fig. 3. It can be seen that the $p_{\text {in }}-t_{\mathrm{d}}$ relation is nonlinear. When $t_{\mathrm{d}}$ is relatively small, its influence is mild. When the treatment time increases from 0 to $30 \mathrm{~min}$, the infiltration pressure is reduced from 22 to $16 \mathrm{MPa}$ by $27 \%$. When the treatment time increases from 30 to $60 \mathrm{~min}$, the infiltration pressure is lowered to 0 by more than $70 \%$. Since the particle size is quite small, the temperature field should reach the equilibrium condition rapidly. For $t_{\mathrm{d}}$ longer than a few min, the $p_{\text {in }}-t_{\mathrm{d}}$ relation should be dominated by the decomposition process. Since a silyl group is attached to the nanopore wall through

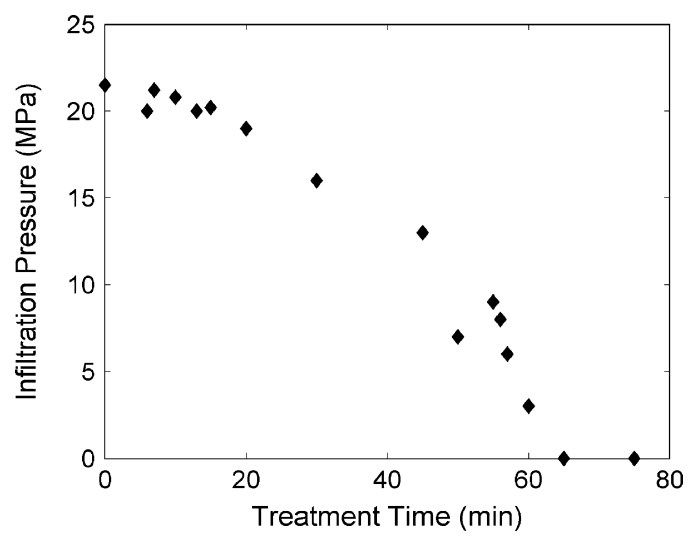

Fig. 3. The infiltration pressure as a function of the decomposition-treatment time.

the oxygen atom, if the $\mathrm{O}-\mathrm{Si}$ bond is broken, further breakage of $\mathrm{Si}-\mathrm{C}$ bond would not affect the surface property. Moreover, the influences of the end group and the side group can be different [14]. Eventually, when the decomposition treatment time is longer than $1 \mathrm{~h}$, most of the silyl groups are deactivated and the nanopore wall becomes hydrophilic. Under this condition, no infiltration of liquid can be observed, since the nanopores are filled immediately after the silica gel is immersed in the liquid phase.

The experimental data also show that the infiltration behavior is dependent on the liquid composition and the testing temperature. When the liquid phase is a saturated sodium chloride solution, as shown in Fig. 2(a), the infiltration pressure increases by about $20 \%$. The profile and the width of infiltration plateau do not vary much, as it should, since the nanopore size distribution and volume are not affected by the addition of electrolyte. The variation in $p_{\text {in }}$ may be attributed to the formation of water molecule clusters around cations. Thus, the influence of silyl groups on the liquid molecules tends to be weaker, the details of which are still under investigation. Similar to the water-based system, after the first loading the confined liquid does not come out of nanopores, and therefore at the second loading no infiltration can take place.

If the infiltration experiment is performed at $90{ }^{\circ} \mathrm{C}$ with water, the sorption isotherm curve is quite similar with the roomtemperature curve of sodium chloride solution. The infiltration pressure increases by about $5 \mathrm{MPa}$, and the variation in infiltration volume is negligible. The increase in $p_{\text {in }}$ can be related to the thermal effect on silyl group. As temperature rises, thermal motion of molecules becomes more pronounced. Thus, the modified solid surface is effectively more nonpolar as the effects of remaining defect sites are reduced, which leads to the increase in degree of hydrophobicity. The "non-outflow" of confined liquid, however, is not affected by temperature.

The effects of addition of electrolyte and increasing temperature on $p_{\text {in }}$ can be superpositioned. As shown in Fig. 2(b), when the silica gel is tested at $90{ }^{\circ} \mathrm{C}$ in saturated sodium chloride solution, the infiltration pressure is around $23 \mathrm{MPa}$, about $7 \mathrm{MPa}$ higher than that of the same silica gel in water at room temperature. The increase in infiltration pressure is close to the summation of the increase caused by sodium chloride and the 
increase caused by temperature change. An even more significant change in system behavior is the liquid defiltration. As the pressure is reduced, before it reaches zero, about $50 \%$ of the confined liquid comes out. As the loading-unloading process is repeated, the empty nanopores can be refilled, leading to the formation of the second infiltration plateau. From the second loading, the profile of sorption isotherm is stabilized. The liquid "outflow" may be related to the increase in solid-liquid interfacial tension, which is the driving force of defiltration.

\section{Concluding remarks}

In the current investigation, it is confirmed through a pressure-induced infiltration experiment that the infiltration pressure of a surface-modified nanoporous silica gel can be adjusted in a broad range by varying the decompositiontreatment time. As the silica gel is heated, the surface group density is lowered and the degree of hydrophobicity varies. The infiltration volume increases slightly. The liquid infiltration and defiltration behaviors are dependent on the liquid composition and the testing temperature. Addition of electrolyte or increasing temperature causes an increase in infiltration pressure; if the two effects are combined, liquid defiltration can be triggered.

\section{Acknowledgement}

This work was supported by The Army Research Office under Grant no. W911NF-05-1-0288.

\section{References}

[1] S. Polarz, B. Smarsly, Nanoporous Materials, J. Nanosci. Nanotechnol. 2 (2002) 581.

[2] A. Han, Y. Qiao, Suppression effect of liquid composition on defiltration of nanofluids in lyophobic environment, J. Phys. D: Appl. Phys. 40 (2007) 3436.

[3] V.K. Punyamurtula, Y. Qiao, Infiltration of pressurized promoter solutions in a mesoporous silica, Microporous Mesoporous Mater. 103 (2007) 35.

[4] J.C. Riviere, S. Myhra, Handbook of Surface and Interface Analysis, CRC Press, 1998.

[5] G. Lu, T. Yu, Energy Absorption of Structures and Materials, CRC Press, 2003.

[6] D. Karami, S. Rohani, Progressive strategies for nanozeolite Y synthesis: a review, Rev. Chem. Eng. 23 (2007) 1.

[7] A. Zukal, Recent trends in the synthesis of nanoporous materials, Chem. Listy 101 (2007) 208.

[8] C.M. Yang, K.J. Chao, Functionalization of molecularly templated mesoporous silica, J. Chin. Chem. Soc. 49 (2002) 883.

[9] A. Han, Y. Qiao, Controlling infiltration pressure of a nanoporous silica gel via surface treatment, Chem. Lett. 36 (2007) 882.

[10] A. Han, Y. Qiao, Effects of surface treatment of a MCM-41 on motions of confined liquids, J. Phys. D: Appl. Phys. 40 (2007) 5743.

[11] M.H. Lim, A. Stein, Comparative studies of grafting and direct syntheses of inorganic-organic hybrid mesoporous materials, Chem. Mater. 11 (1999) 3285.

[12] Y. Qiao, X. Kong, Effective dewetting in a microporous particle, J. Fluids Eng-Trans. ASME 127 (2005) 1128.

[13] J.A. Fay, Fluid Mechanics, MIT Press, 1994.

[14] Y. Qiao, A. Han, Effects of nanopore size on properties of modified inner surfaces. Langmuir, in press. 